\title{
So fern und doch so nah ...
}

Die Pandemie hat die Büros geleert. Ganze Firmen wechselten innerhalb kürzester Zeit zum Arbeiten von zu Hause oder ins Homeoffice, wie man hierzulande gern sagt. Damit hat das Arbeiten von außerhalb - die sogenannte „Remote Work“ einen unerwarteten und starken Schub erhalten. Groß waren zuvor die Ängste und Befürchtungen in Personalabteilungen und im Management. So mancher dachte, sein Unternehmen werde zusammenbrechen, wenn kaum noch jemand ins Büro kommt. Stattdessen funktionierten viele Betriebe nach kurzer Eingewöhnungszeit so gut wie vorher.

Wie war das möglich? Ein wesentlicher Grund dürfte gewesen sein, dass die für ein effizientes Arbeiten von außerhalb nötige Technik bereits seit einigen Jahren in vielen Unternehmen vorhanden war - sie wurde lediglich wenig genutzt. Arbeitsplätze waren mit Webcams und Headsets ausgestattet, Videokonferenzsoftware und Chatprogramme auf den Rechnern installiert. Benutzt wurden diese Werkzeuge aber oft nur sporadisch, von einigen gar nicht. Gewohnheit ist ein starkes Hemmnis für Veränderung. Neues erscheint vielen erst mal bedrohlich, besser, man macht alles auf die „bewährte“ Art, glauben viele. Das ist nicht immer falsch. Verändern, nur um des Veränderns willen, ergibt keinen Sinn, sondern kostet unnötig Energie und birgt das Risiko, gut eingespielte Prozesse zu (zer)stören.

Um jeden Preis am Althergebrachten festzuhalten, ist aber ebenfalls nicht gut, oft sogar gefährlich. Ohne ein Umschwenken auf Remote Work wären 2020 und 2021 sehr viele Unternehmen wirtschaftlich in Schieflage geraten, das konnte oft verhindert werden. Jetzt, wo das Ende der Pandemie absehbar ist, sollte man keinesfalls einfach versuchen, zum Status quo ante - zur "guten alten Zeit“" zurückzukehren. Stattdessen gilt es, eine neue Balance zu finden, zwischen der Arbeit mit Kollegen vor Ort und der von außerhalb. Nicht jede Besprechung muss vor Ort stattfinden. Gleichzeitig hat uns die Zeit, in der es nur via Videokonferenz ging, aber auch gezeigt, dass es ganz ohne echte persönliche Treffen auch nicht geht. Wie so oft braucht es das richtige Werkzeug für die Aufgabe. Der Werkzeugkasten hat sich erweitert, dass wir nun nicht mehr nur einen Hammer haben, sondern auch noch Zange und Schraubendreher bedeutet nicht, dass der Hammer überflüs-

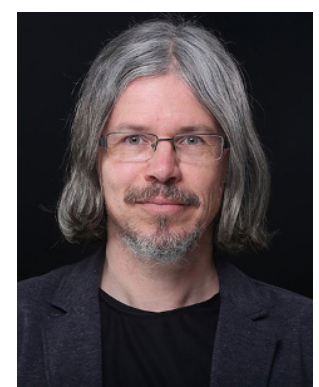

Peter Pagel $(\triangle)$

peter.pagel@springer.com

Wiesbaden, Deutschland

sig wird. Aber, um eine Schraube in die Wand zu bekommen, ist Letzterer nicht das beste Werkzeug.

Ich wünsche Ihnen, dass Sie möglichst oft das am besten geeignete Werkzeug wählen.

Peter Pagel

Chefredakteur

Wirtschaftsinformatik \& Management 2021 • 13 (6): 401

https://doi.org/10.1365/s35764-021-00371-6

Angenommen: 12. Oktober 2021

(c) Springer Fachmedien Wiesbaden GmbH, ein Teil von Springer Nature 2021 